\title{
SIM Configuration Evolution
}

\author{
Dr. Kim M. Aaron \\ California Institute of Technology \\ Jet Propulsion Laboratory \\ 4800 Oak Grove Dr. 301-486 \\ Pasadena, CA 91109 \\ 818-354-2816 \\ Kim.M.Aaron@ipl.nasa.gov
}

\begin{abstract}
Abstract - The Space Interferometry Mission (SIM) is a space-based $10 \mathrm{~m}$ baseline Michelson interferometer. Planned for launch in 2005 aboard a Delta III launch vehicle, or equivalent, its primary objective is to measure the positions of stars and other celestial objects with an unprecedented accuracy of 4 micro arc seconds. With such an instrument, tremendous advancement can be expected in our understanding of stellar and galactic dynamics. Using triangulation from opposite sides of the orbit around the sun (i.e. by using parallax) one can measure the distance to any observable object in our galaxy. By directly measuring the orbital wobble of nearby stars, the mass and orbit of planets can be determined over a wide range of parameters. The distribution of velocity within nearby galaxies will be measurable. Observations of these and other objects will improve the calibration of distance estimators by more than an order of magnitude. This will permit a much better determination of the Hubble Constant as well as improving our overall understanding of the evolution of the universe.
\end{abstract}

SIM has undergone several transformations, especially over the past year and a half since the start of Phase A. During this phase of a project, it is desirable to perform systemlevel trade studies, so the substantial evolution of the design that has occurred is quite appropriate. Part of the trade-off process has addressed two major underlying architectures: SIM Classic; and Son of SIM. The difference between these two architectures is related to the overall arrangement of the optical elements and the associated metrology system. Several different configurations have been developed for each architecture. Each configuration is the result of design choices that are influenced by many competing considerations. Some of the more important aspects will be discussed.

The Space Interferometry Mission has some extremely challenging goals: millikelvin thermal stability, nanometer stabilization of optics, picometer measurement of wavefront, and others. In order to meet these goals, a significant amount of technological development is required. Although there has been a program operating for about a decade developing technologies specifically to address the challenges of space-based interferometry, there still remains a tremendous effort to achieve the incredible accuracy required of SIM. The projected viability of some of these areas has influenced design choices during the evolution of the many configurations that have been developed. For instance, the perceived complexity of the IR laser metrology system used to measure and control the positions of key optical elements was the strongest discriminator between the two architectures, and led to a decision to select SOS rather than Classic in early 1998. More recently, an appreciation of the sensitivity to beam-walk within the SOS architecture is forcing a reconsideration of that decision. At the time of submission of this abstract, there is some hope that a fullaperture metrology system may alleviate this issue.

In addition to describing the current configuration of SIM, the influence of a few selected areas on the evolution of the configuration will be discussed.

\section{TABLE OF CONTENTS}

1. SIM OVER VIEW

2. SIM ARCHITECTURES

3. SIM CLASSIC DESCRIPTION

4. SON OF SIM DESCRIPTION

5. COMPARISON OF THE TWO ARCHITCETURES

6. SUMMARY

\section{SIM OVERVIEW}

The Space Interferometry Mission will launch a Michelson interferometer with a $10 \mathrm{~m}$ baseline into a heliocentric IAU orbit aboard a Delta III or equivalent launch vehicle in 2005. The Jet Propulsion Laboratory, Lockheed Martin, and TRW are developing SIM for NASA under a collaborative effort. TRW will develop the precision structure, the engineering subsystems and bus, and will perform the overall integration and test. LM is responsible for the instrument. JPL provides overall coordination and interferometry expertise.

The primary objective of SIM is to measure the positions of stars and other celestial objects to a precision of about $4 \mu$ arc second ( $\mu$ as, about a billionth of a degree). This capability allows scientists to infer the existence of planets orbiting other stars by directly measuring the motion of the star. In addition to being able to detect smaller planets than current techniques, SIM also eliminates ambiguity about the 
-iaclination of the orbits of planets. Current techniques provide only an upper bound on planet mass. Using parallax (triangulation from points in the orbit at opposite sides of the sun) one can measure the distance to any star in our galaxy observable by SIM (visible stars brighter than $18^{\text {th }}$ magnitude). In effect, this permits calibration of various distance estimators currently used that correlate distance with other measurements, such as brightness. Improved distance estimators will enable more precise estimates of the Hubble constant and the age of the universe. SIM will be able to measure the distribution of rotational velocity within nearby galaxies. This will improve estimates of the masses of these galaxies, improving our understanding of galactic dynamics and evolution. If conditions are favorable SIM will be able to measure deflection of starlight by postulated dark matter.

The project is in Phase A (Conceptual Design). During this phase of a project, many system-level tradeoffs are typically performed. SIM is certainly no exception. SIM is considering two significantly different architectures and several configurations have been developed for each over the past couple of years. The two architectures are SIM Classic and Son of SIM (SOS). The two architectures will be contrasted and some of the pros and cons will be discussed. The project is developing layouts for both architectures, leading to a decision by November 1999.

\section{What is an interferometer?}

An interferometer is an optical instrument that uses two or more telescopes to collect light from a single target and combine the light coherently on a detector so that interference fringes form. For a white light interferometer, such as SIM, a strong central fringe forms only when the light from both arms is completely in phase. That is, the total pathlength from the star to the detector is identical for both arms. SIM uses two telescopes separated by $10 \mathrm{~m}$ or more. The main collector mirror in each telescope is about $35 \mathrm{~cm}$ in diameter. The physical separation of the two collector telescopes is referred to as the physical baseline. For SIM, the planned baseline is at least $10 \mathrm{~m}$. The pathlength the light follows within each arm is adjusted until the two beams are in phase, as indicated by the maximum constructive interference fringe on the detector. This adjustment is made to within a small fraction of the wavelength of light. By simultaneously measuring the geometry of the interferometer using incredibly precise laser interferometers (precision of a few tens of picometers), one can determine the angle between the physical baseline and the direction to the star.
Although SIM can measure the angle between its baseline and a target with a precision of $7.5 \mu$ as (single measurement accuracy) there is no existing reference frame to that accuracy. One of the first tasks SIM will perform is to create a self-consistent grid of guide stars. Relative angle will be measured between many pairs of stars. Various calibration parameters are then adjusted to make these measurements self-consistent. We refer to this as "closing the grid." This process will be a sort of least squares fit solution to many simultaneous non-linear equations, probably with more equations than unknowns. Once the positions of several hundred reference stars have been determined in this way, SIM will measure the positions of science stars and other targets of interest with respect to this grid.

Existing star trackers used for traditional spacecraft attitude determination are completely inadequate for determining the attitude of SIM's baseline to the required precision of a few $\mu$ as. Basically, one would need a star tracker with a $10 \mathrm{~m}$ aperture to match the resolution of SIM. Instead, SIM uses two additional, essentially identical, interferometers to measure the orientation of the physical baseline with respect to the grid stars. Each guide interferometer measures the angle between the baseline and a convenient grid guide star. Provided one selects appropriate guide stars (not a degenerate geometry), the orientation can be determined to the required single measurement accuracy of $7.5 \mu$ as. To achieve the overall required accuracy of $4 \mu \mathrm{as}$, it is necessary to take several independent measurements of each target and average the results to beat down the uncorrelated noise contributions.

\section{Fiducuials and the Baseline}

The term, "baseline" has two related but distinct meanings. Two parallel tubes of light coming from the target star are collected by primary mirrors. These two tubes of light are then manipulated by several successive optics (mostly reflective; there is one beam splitter) in such a way that the tubes are combined on a detector. In SIM (either architecture) the tubes of collected light have a diameter of $33 \mathrm{~cm}$ and by the time they reach the detector they have a diameter of $3 \mathrm{~cm}$. Starting at a single point on the detector and tracing a single ray back out through the system, it will split into two rays, one for each arm. Outside of the interferometer, these two rays should be parallel if the optics are aligned correctly. The perpendicular (minimum) distance between these two incoming rays is the astrometric baseline. For perfect optics, the astrometric baseline would be the same for all points on the detector. 


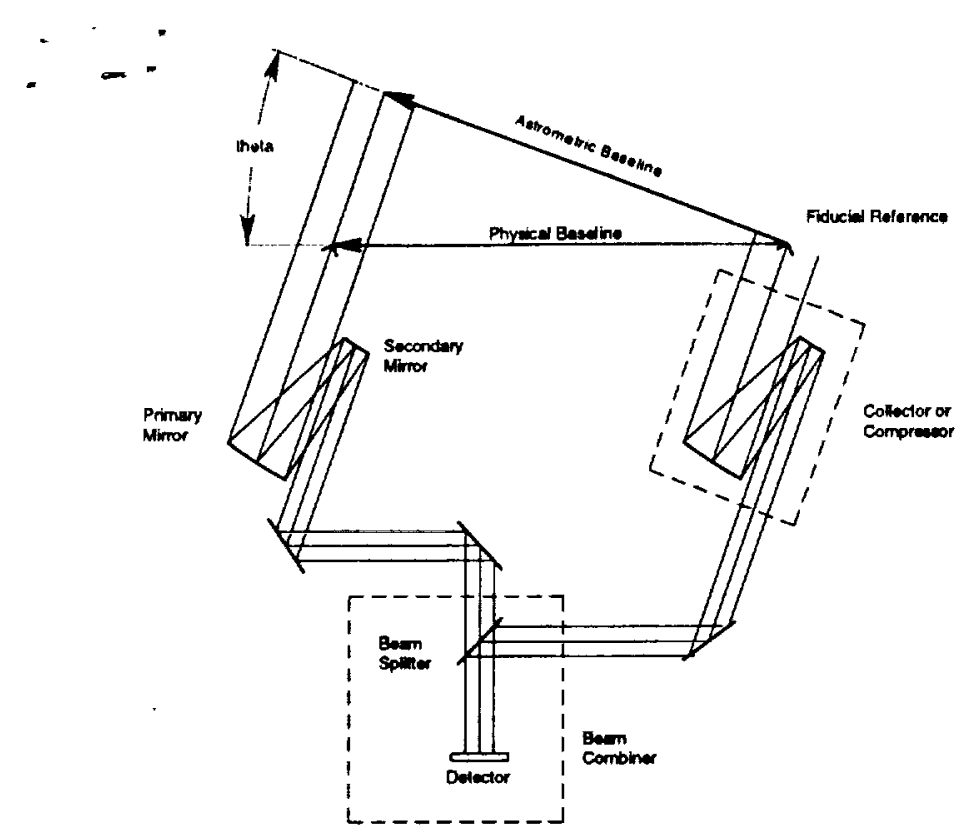

Figure 1 Schematic defining Physical and Astrometric Baselines

In SIM (both architectures), a reference point, or fiducial, is installed in the field of view of each collector. On SOS, this fiducial is suspended in front of the collector as shown in Fig 1. On SOS, the collector moves to change the line of sight. On Classic, the fiducial is physically mounted on the surface of the siderostat mirror (shown in Fig. 4) which aims the line of sight of the collector. Both designs use retroreflecting corner cubes as the fiducial, although other choices have been considered, such as hemispherical mirrors.

Various auxiliary cameras, detectors and beacons are used to actively control the positions of various optical elements in the light train to keep the fiducial in the center of the field of view of each collector, and also to ensure that a hypothetical incoming ray passing through the fiducials would end up at the same point on the beam combiner detector. Again assuming ideal optics, the field of view from each arm of the interferometer will be coincident on the detector and all points will have the same astrometric baseline. Since we rely on the active control to maintain the alignment of the two optical trains on the two fiducials, we can measure the physical distance between the two fiducials to infer the astrometric baseline. The physical baseline is the vector connecting one fiducial to the other. Usually, this vector will not be perpendicular to the incoming starlight, but this angle is precisely what is measured by the interferometer, so the astrometric baseline is simply the physical baseline multiplied by the cosine of the angle between the starlight and the normal to the physical baseline:

Astrometric Baseline $=$ Physical Baseline $\times \cos (\theta)$.
Corrections and Second Order Effects

When measuring the positions of stars to a precision of 4 $\mu a s$, it is necessary to account for many effects that are normally negligible. For instance, the gravitational pull of the sun bends the light from distant sources several thousand $\mu$ as depending on the direction of the line of sight. If one is looking exactly anti-sunward, then there is no bending. For light passing near planets in our solar system at the time of observation, especially Jupiter, it is necessary to correct for this gravitational bending also. In fact, gravitational lensing may allow SIM to infer the existence of concentrated planet sized masses in interstellar space if one happens to pass between SIM and a target. SIM will revisit target several times throughout its five year mission (to boldly go...). Apparent motion of a star with the right "signature" will be indisputable evidence of the existence of such dark matter objects.

For SIM Classic, the three interferometers (nominally two guide interferometers and one science interferometer) are essentially operating independently. External metrology laser gauges are used to measure the orientation of the three baselines and feedback is used to maintain the three baselines parallel. Even though the residual positioning errors are very small (baselines angles differ by a few milli arc seconds) the trigonometric corrections cannot use the typical small angle approximations ( $\sin \theta \approx \theta$, etc.). It is necessary to use higher order approximations since the second order and even third order terms are not negligible.

As the look-direction of SIM changes with respect to its own velocity, the apparent angle to "stationary" distant objects will change due to relativistic effects. To correct for this, it is necessary to know the velocity of SIM with a precision of a few millimeters per second. It is possible to achieve this level, but it is challenging to do so.

\section{SIM ARCHITECTURES}

Two basic conceptual architectures for SIM (SIM Classic, and SOS: Son of SIM) have been under consideration for some time. These are distinguished by the arrangement of the optical elements to form the three baselines. Within each architecture, several configurations have been developed to assess the feasibility of the two architectures.

\section{SIM CLASSIC DESCRIPTION}

SIM Classic is configured as a Tee-shaped structure when deployed. Along the arms of the tee are distributed seven collector bays (or siderostat bays). Within each bay is a fixed $11: 1$ compressor comprised of a $33 \mathrm{~cm}$ diameter clear 
-aperture primary mirror and a $3 \mathrm{~cm}$ secondary. The mirrors are off-axis confocal paraboloids. Parallel star light entering the compressor exits parallel but compressed down to a $3 \mathrm{~cm}$ diameter bundle. These compressor assemblies are essentially fixed in the geometry of the layout. Facing each compressor is a gimbaled flat siderostat mirror which serves to aim the system at targets of interest. The range of motion of the siderostats is $\pm 4.75^{\circ}$ corresponding to a motion of the line of sight of $\pm 7.5^{\circ}$. For a given space system attitude, any star within a $15^{\circ}$ cone centered on the nominal line of sight can be selected. This cone is referred to as the field of regard (FOR). The actual field of view (FOV) is a few tens of milli arc seconds. Each of the seven siderostat bays is lined up with one another such that they all point nominally perpendicular to the arms of the tee. Thus, all seven share the same FOR. In the most recent incarnation, the center of this FOR was elevated up $30^{\circ}$ from the top surface of the tee. For earlier layouts, this angle was $45^{\circ}$ or $90^{\circ}$.

In the stem of the tee, there is a switchyard of mirrors which can select the starlight from any pair of collectors and direct the two beams onto any one of four beam combiners. Thus any pair of collectors can be used as an interferometer with baseline equal to the spacing between the two. The spacings of the seven assemblies along the tee were selected in such a way that no two combinations would yield the same baseline. The minimum baseline is about $0.4 \mathrm{~m}$ whereas the maximum is $10 \mathrm{~m}$. With seven elements, it is possible to form 21 different pairs, each with its own baseline length. These values are reasonably uniformly distributed between the minimum and maximum values so there are no substantial gaps in the $u-v$ plane for imaging. Only three interferometers are needed to make a measurement.

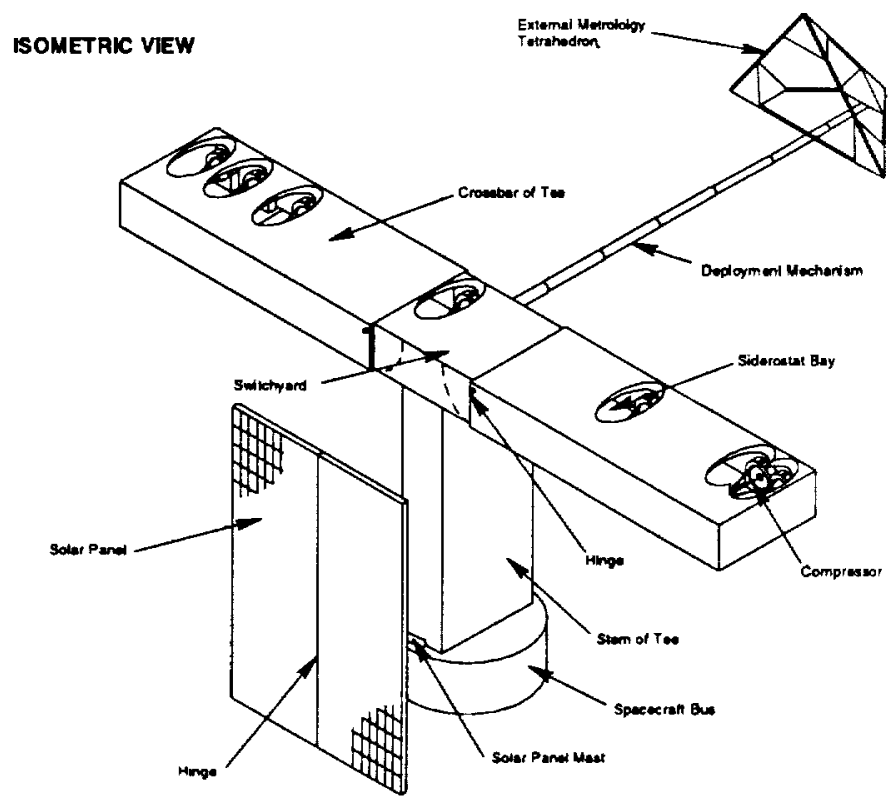

Figure 2 SIM Classic Deployed Configuration
Fin any parlicular observation, it is necessary to have three incerformelers operalling: two lo ate ats guides and one to measure the science larget. With Clatsic, astrometric measurements of the science target are made using the maximum hascline of achicve the greatcst precision. However, this implies that the suicles must use smaller batselines. This geometric disadvantage is offect by the brightness of the guide stars and is not a great issue.

\section{Aligning the Corner Cubes: Extomal Metrology}

The purpose of the guide interterometers is to establish the orientation of the science bascline. Since each of the guide interferometers has its own baseline, really the guides determine very precisely one angle from a well-known guide star to that baseline. In order to establish the required orientation of the science baseline, it is necessary to determine the relative orientations of the three baselines.

This is accomplished by using the external metrology system. The system uses 28 interferometric laser gauges to measure the distances between the seven fiducials mounted on the siderostats and each of four fiducials mounted on a separate external metrology tetrahedron. In addition, there are six laser gauges to measure the distances between each of the vertices of the tetrahedron. Using all this geometric information, it is possible to solve for the orientation of the science baseline.

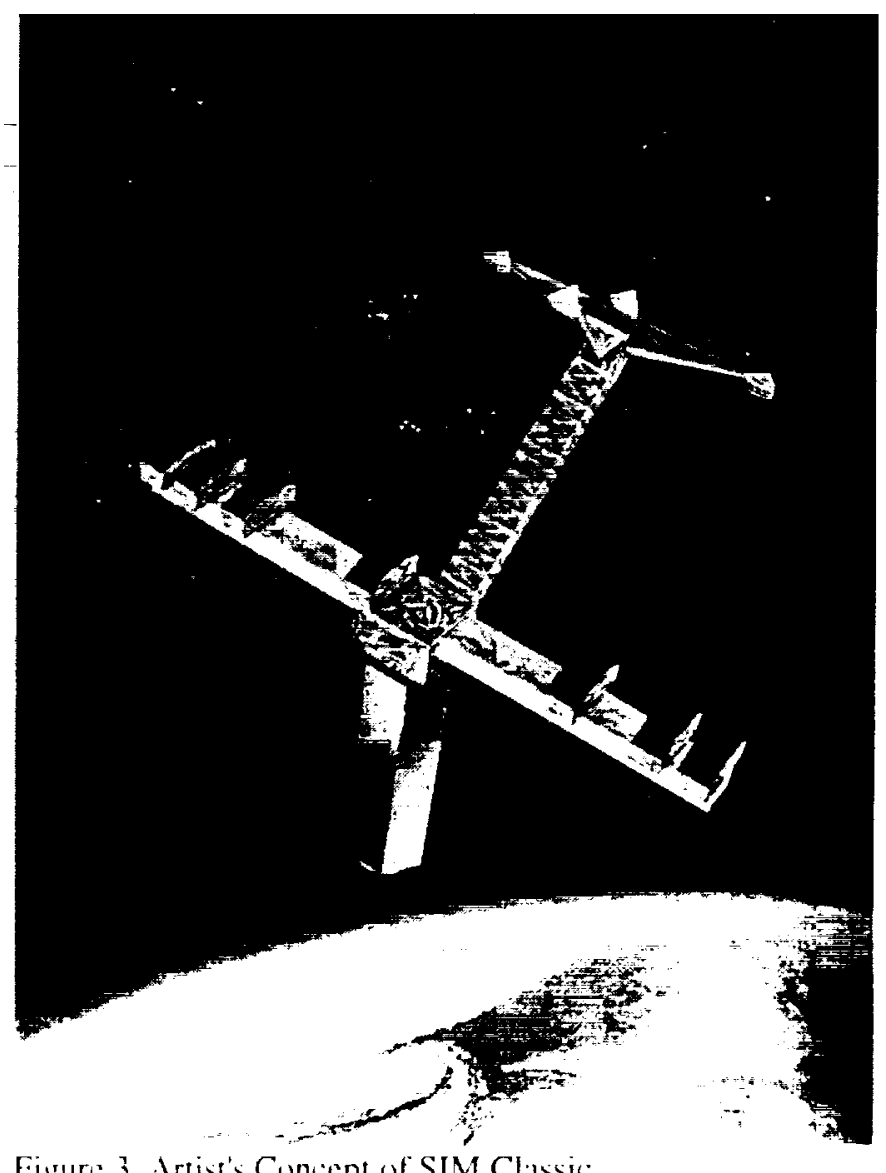




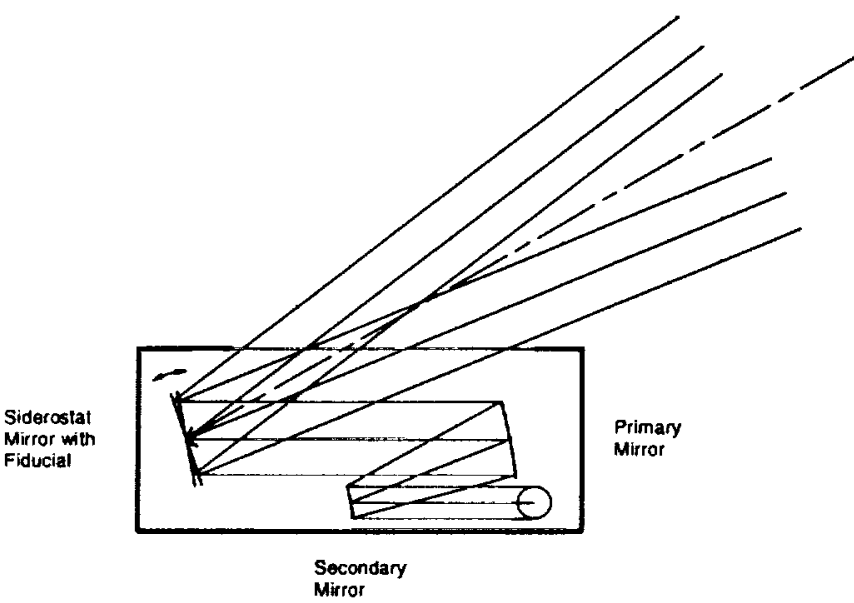

Figure 4 SIM Classic Siderostat Bay

\section{SON OF SIM DESCRIPTION}

Chronologically, the Son of SIM architecture followed SIM Classic. The key distinguishing factor between the two architectures is way in which the fiducials for the guide interferometers are related to the fiducials for the science (astrometric) interferometer. For SOS, there are only two fiducials and all collectors share the same fiducials. The same fiducials are used for both science and guide interferometers.

The Son of SIM configuration includes two collector pods, each of which houses four collector assemblies. Each collector is used in conjunction with an essentially identical collector in the other collector pod. In the current configuration, one of these pods is fixed, whereas the other moves on precise rails to vary the distance between the two pods. Alternative configurations have had two moving pods, and present work is leaning towards reverting to two moving pods. However, this is not an essential difference for purposes of comparing SIM Classic and SOS.

As stated earlier, only three interferometers are required: two to act as guide interferometers to establish the orientation of the physical baseline, and a third to measure the position of the science target with respect to that baseline. The fourth is included for redundancy. This extra interferometer is used primarily to achieve small baselines to satisfy the imaging objectives. However, this fourth interferometer will be adequate to perform the function of guide interferometer in the unlikely event that some unexpected failure should prevent one of the other three interferometers from working. Similarly, the nominal guide interferometers can also perform the function of the science interferometer.

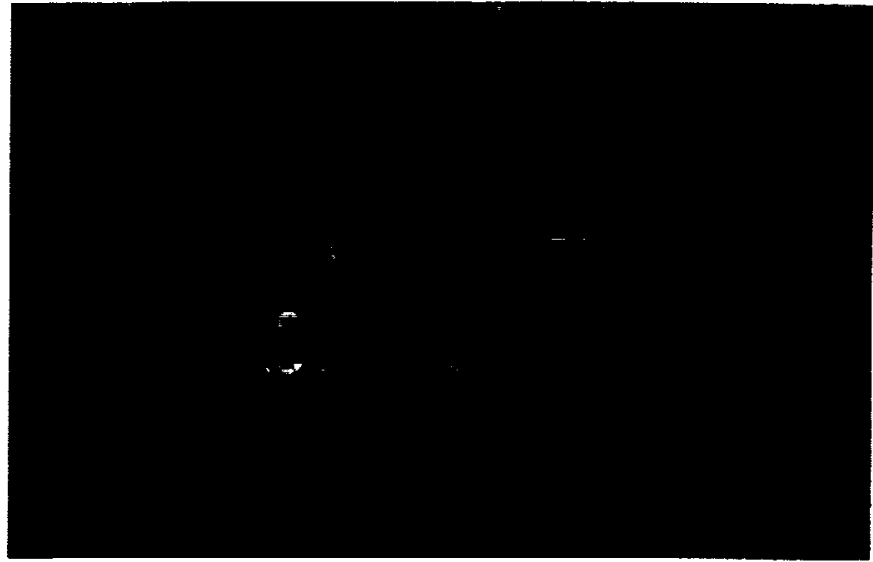

Figure 5 Artist's Concept of Son of SIM

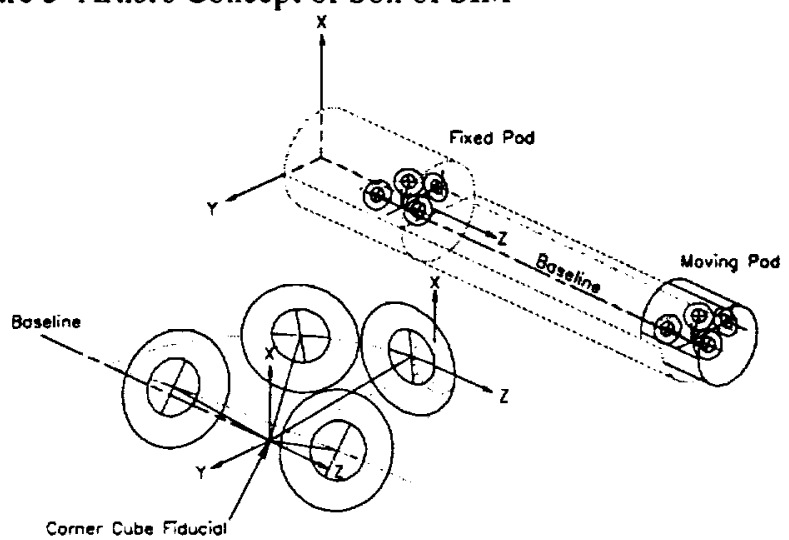

Figure 6 SOS Layout with enlarged view of collectors indicating their range of motion

As illustrated in Fig 6, since all four collectors share a common fiducial at the center of their fields of view, it is not possible for them to be pointed in the same direction, at least at the same time. Each collector assembly is moved as a unit such that it remains tangent to a hypothetical spherical surface centered at the corner cube fiducial. The same field of regard is maintain $\left(15^{\circ}\right.$ cone) as for Classic. In Fig. 6 the four smaller circles represent the physical size of the mirror. The large circles represent the space covered by the mirrors as they move about their fields of regard. It can be seen that the collectors will not collide at the extremes of their motion. However, concepts have been investigated in which overlapping fields of regard have been allowed

\section{Measuring the Baseline}

Since all four interferometers share the same two fiducials, it is not necessary to have the external metrology tetrahedron that Classic has. Instead, a single interferometric laser gauge is used to measure the distance between the two corner cubes. This information, coupled with the knowledge of the angles between the two guide stars and the single baseline is sufficient to determine the baseline vector. 


\section{COMPARISON OF THE TWO ARCHITECTURES}

In comparing two different architectures, it is difficult to separate the particular point designs from the inherent differences in the architecture. A comparison of two point designs may be invalid if the differences are due to essentially random particular implementation choices of the design team at the time. A true comparison of two architectures should focus on the inherent differences between the two architectures. This comparison validly should include implications about the ease of design associated with the choice, however.

In comparing SIM Classic and Son of SIM, the important difference between the two architectures is the fiducial defining the baselines. SOS inherently uses the same two fiducials for all four interferometers. Classic uses seven independent fiducials (only six are used at a given time). It is therefore necessary to add some means of measuring the relative orientations of the three separate baselines. This led to the external metrology tetrahedron (a particular design choice). The inherent difference in the architectures is the need to resolve the three baselines. This particular point was indeed the strongest discriminator that finally led to the selection of the SOS architecture over the Classic. There are, however, a host of other differences.

One such inherent difference between the two architectures is that for SOS, with four collectors sharing a single fiducial, it is not possible for the collectors to look the same direction at the same time. With Classic, it is possible for all three interferometers to look the same direction simultaneously. Actually, it is not of much use for all three interferometers to look at the same target, but the guide interferometers can use reference stars much closer to the target star. It is not clear that the larger angles between reference stars and science stars for SOS will reduce the precision however, but the additional constraint does make the geometric layout of the collectors more complicated for SOS.

On the other hand, for SOS, the collectors are forced to be close together, which to some extent simplifies the layout of the rest of the flight system. Although it is not a very clear advantage, there does appear to be somewhat more overall configuration layout freedom in the SOS architecture. Since Classic tends to drive the design towards fixed collectors, this then constrains the collectors to be arranged over a fairly large physical extent. This provides less freedom to layout the geometry within the fairly tight volume of the launch vehicle fairing. On the other hand, the fairly large pods of SOS impose a different difficulty in this process of laying out the system to fit inside the fairing. The relatively large radial extent leaves less room to place structure around the pods.

To be fair, it was very challenging to find solutions for both layouts. When the layouts shown in the figures above were initially conceived, the launch vehicle was a Delta II 7920. Recently, the project made a different architectural choice. It was decided to avoid the difficulties of operating an observatory in low earth orbit and instead to use an earthescape heliocentric orbit. This requires a larger, albeit more expensive, launch vehicle (Delta III class) which happens to have a larger fairing. This change greatly eases the difficulties of packaging a 10 meter class precise structure.

This decision also simplifies many other aspects of the mission, such as earth and moon avoidance, solar power collection, attitude control, sun baffle design. Now that a larger fairing is available, the layout of either Classic or SOS would be eased, and so the relative merit of this geometric size issue is reduced now.

When it is necessary to change the baseline, as in imaging, then Classic must use its switchyard mirrors to reconfigure the various collector pairs to form new interferometers. This means it is necessary to lose lock on at least one star at a time during the process. For SOS, there is a chance that the interferometers can remain operating and locked onto their stars while the pod of collectors is moved. This is not essential, and would increase the precision required of the trolley system, but it would enable an increase in observational efficiency since it would eliminate the time required to reacquire stars. This is not likely to be a major consideration since the overall time spent acquiring stars is a small fraction of the total observational time. However, it is a small advantage for SOS in that it allows some additional design freedom.

The decoupled fiducials for Classic makes it possible to achieve the variable baseline required for imaging without the need to move the collectors laterally. The u-v plane is filled reasonably uniformly using the twenty-one combinations of the seven fixed collectors. SOS basically is driven towards a solution that requires the movement of at least one large assembly of four collectors. Although a design concept has been developed to achieve this, the implementation is likely to be complicated.

With the moving trolley needed for SOS, it was quite challenging to achieve very small baselines $(0.5 \mathrm{~m})$. The difficulty arose since the physical size of the pod size to house four collectors is on the order of $2 \mathrm{~m}$ in linear dimension. It isn't very feasible to move the two collector pods closer than a physical separation of $2 \mathrm{~m}$. In order to achieve $0.5 \mathrm{~m}$ astrometric baseline, it was necessary to include a collector that could be aimed only 15 degrees away from the physical baseline. Although this obstacle now appears to have been overcome, it is an additional constraint in the already complicated layout of four collectors in a pod. As the design evolves to meet challenges not yet recognized, this factor is a negative aspect. For Classic, it is quite easy (in comparison) to achieve small baseline. One simply places two of the 
$=$ collectors side by side, limited only by the physical size of the mirrors and their mounting means.

Another constraint favoring Classic is the freedom to point the line of sight independent of the translation. For SOS, the collectors have to translate very precisely over a range of about $30 \mathrm{~cm}$ in order to keep the center of the line of sight aimed at the fiducial whenever the line of sight must be tilted. Although Classic does need some translational stages to maintain alignment, the tilt is essentially independent of the translation. Classic can aim its collectors by simply tilting them (or as selected for the particular point design, by a tip-tilt mechanism with a flat siderostat mirror). The SOS design choice is a hexapod consisting of six linear actuators with rather stringent precision requirements over a range of motion of many centimeters. Other options are available, but still, it is basically inherent in the architecture that this pointing aspect is easier to implement for Classic.

The layout of the configuration of a space vehicle is affected by very many factors. Just a few have been mentioned here. The trade study that was performed to ultimately choose between the two architectures described here, SIM Classic vs Son of SIM, took several months, culminating in a twoday long review to a very experienced panel of scientists, engineers, and managers. The decision was made considering the viewpoints of all of the above and was a very difficult decision. Had there been a blatantly obvious difference between the two, the decision would have been much easier. In fact, it probably would not have required the two day review, nor such a protracted investigation. However, at the end of the two days, and after hours of debate, the decision was made in favor of SOS. Although there were presentations from many areas, for the most part, there were no overwhelmingly compelling reasons to select one versus the other. The single major exception was the external metrology system required for Classic. The assembly of thirty-four precision laser interferometer gauges stuck out literally like a sore thumb. It is clear that this single factor ultimately tilted the scale in favor of SOS.

\section{SUMMARY}

A brief description has been presented of two architectures, each of which could achieve the science objectives of the Space Interferometry Mission, SIM. Point designs for both architectures have been contrasted and some of the pros and cons have been discussed. It is not feasible to create a comprehensive list of all the differences, nor would it be helpful. The main point has been to illustrate that there are many factors that can influence the choice between two fairly attractive options. The rationale for the selection of SOS over Classic has been explained, at least partially. The decision was difficult, but having made it, the SIM project can now move ahead with renewed vigor on a challenging but rewarding course towards a successful mission.

\section{BIOGRAPHY}

Dr. Aaron received his M.Sc and $\mathrm{PhD}$ in Aeronautics from Caltech in 1980 and 1985 respectively and a B. Engineering (Honors Mechanical) from McGill University in Montreal, Canada in 1979. Since graduating, he has worked at the Jet Propulsion Laboratory on a wide range of projects. He has been involved in the development of the mechanical configuration of spacecraft for about thirty different space missions in the conceptual phase.

\section{ACKNOWLEDEMENT}

This work was carried out by the Jet Propulsion Laboratory, California Institute of Technology under a contract with the National Aeronautics and Space Administration. 